\title{
Using Green Biosynthesized Lycopene-Coated Selenium Nanoparticles to Rescue Renal Damage in Glycerol-Induced Acute Kidney Injury in Rats
}

\author{
Ashraf Al-Brakati' \\ Khalaf F Alsharif ${ }^{2}$ \\ Khalid J Alzahrani (iD) ${ }^{2}$ \\ Saeed $\mathrm{Kabrah}^{3}$ \\ Osama Al-Amer ${ }^{4,5}$ \\ Atif Abdulwahab Oyouni $\mathbb{D}^{5,6}$ \\ Ola A Habotta ${ }^{7}{ }^{7}$ \\ Maha S Lokman (1D) 8,9 \\ Amira A Bauomy ${ }^{10}$ \\ Rami B Kassab (D, ${ }^{9,1}$ \\ Ahmed E Abdel Moneim (1D ${ }^{9}$ \\ 'Department of Human Anatomy, College of \\ Medicine, Taif University, Taif, 21944, Saudi \\ Arabia; ${ }^{2}$ Department of Clinical Laboratory \\ Sciences, College of Applied Medical \\ Sciences, Taif University, Taif, 21944, Saudi \\ Arabia; ${ }^{3}$ Department of Laboratory \\ Medicine, Faculty of Applied Medical \\ Sciences, Umm AIQura University, Makkah, \\ Saudi Arabia; ${ }^{4}$ Department of Medical \\ Laboratory Technology, Faculty of Applied \\ Medical Sciences, University of Tabuk, Tabuk, \\ Saudi Arabia; ${ }^{5}$ Genome and Biotechnology \\ Unit, Faculty of Sciences, University of Tabuk, \\ Tabuk, Saudi Arabia; ${ }^{6}$ Department of Biology, \\ Faculty of Applied Medical Sciences, \\ University of Tabuk, Tabuk, Saudi Arabia; \\ ${ }^{7}$ Department of Forensic Medicine and \\ Toxicology, Faculty of Veterinary Medicine, \\ Mansoura University, Mansoura, Egypt; \\ ${ }^{8}$ Biology Department, College of Science \\ and Humanities, Prince Sattam bin Abdul \\ Aziz University, Alkharj, Saudi Arabia; \\ 'Department of Zoology and Entomology, \\ Faculty of Science, Helwan University, Cairo, \\ Egypt; ${ }^{10}$ Department of Science \\ Laboratories, College of Science and Arts, \\ Qassim University, ArRass, 52719, Saudi \\ Arabia; " 'Department of Biology, Faculty of \\ Science and Arts, Al Baha University, \\ Almakhwah, Al Baha, Saudi Arabia
}

Correspondence: Ahmed E Abdel Moneim Email aest1977@hotmail.com
Purpose: Selenium nanoparticles (SeNPs) have recently gained much attention in nanomedicine applications owing to their unique biological properties. Biosynthesis of SeNPs using nutraceuticals as lycopene (LYC) maximizes their stability and bioactivities. In this context, this study aimed to elucidate the renoprotective activity of SeNPs coated with LYC (LYCSeNPs) in the acute kidney injury (AKI) model.

Methods: Rats were divided into six groups: control, AKI (glycerol-treated), AKI+sodium selenite $\left(\mathrm{Na}_{2} \mathrm{SeO}_{3} ; 0.5 \mathrm{mg} / \mathrm{kg}\right), \mathrm{AKI}+\mathrm{LYC}(10 \mathrm{mg} / \mathrm{kg}), \mathrm{AKI}+\mathrm{LYC}-\mathrm{SeNPs}(0.5 \mathrm{mg} / \mathrm{kg})$ and treated for 14 days.

Results: Glycerol treatment evoked significant increases in rhabdomyolysis-related markers (creatine kinase and LDH). Furthermore, relative kidney weight, Kim-1, neutrophil gelatinase-associated lipocalin (NGAL), serum urea, and creatinine in the AKI group were elevated. Glycerol-injected rats displayed declines in reduced glutathione level, and superoxide dismutase, catalase, glutathione peroxidase, and glutathione reductase activities, paralleled with downregulations in Nfe2l2 and Hmox-1 expressions and high renal MDA and NO contents. Glycerol-induced renal inflammation was evident by rises in TNF- $\alpha$, IL-1 $\beta$, IL-6, and upregulated Nos 2 expression. Also, apoptotic (elevated caspase3, Bax, and cytochrome-c with lowered Bcl-2) and necroptotic (elevated Pipk3 expression) changes were reported in damaged renal tissue. Co-treatment with $\mathrm{Na}_{2} \mathrm{SeO}_{3}, \mathrm{LYC}$, or LYC-SeNPs restored the biochemical, molecular, and histological alterations in AKI. In comparison with $\mathrm{Na}_{2} \mathrm{SeO}_{3}$ or LYC treatment, LYC-SeNPs had the best nephroprotective profile.

Conclusion: Our findings authentically revealed that LYC-SeNPs co-administration could be a prospective candidate against AKI-mediated renal damage via antioxidant, antiinflammatory, anti-apoptotic and anti-necroptotic activities.

Keywords: acute kidney injury, apoptosis, inflammation, lycopene, selenium nanoparticles, oxidative stress, necroptosis

\section{Introduction}

Acute kidney injury (AKI) is a severe clinical condition of abrupt kidney dysfunction associated with structural damage and failure to maintain the acid-base balance or eliminate waste products. ${ }^{1}$ Many aetiological factors are involved in the pathophysiology of AKI, including renal ischemia, nephrotoxic drugs, endogenous toxins, or infections. ${ }^{2,3}$ It was reported that the loss of renal function had been reversed in most survived patients. However, the deaths are still high (over 50\%). ${ }^{4}$ To date, 
there are no efficient therapies to prevent or accelerate the recovery from AKI. Hence, the search for an effective AKI therapy has gained much attention. ${ }^{5}$

Intramuscular glycerol injection evokes AKI through induction of rhabdomyolysis (RM). ${ }^{3}$ This experimental model is commonly used for understanding the mechanisms of renal injury. The extensive breakdown of skeletal muscles generates a high amount of myoglobin, surpassing plasma protein-binding capacity, resulting in glomerular filtration and tubular reabsorption of myoglobin. Myoglobin precipitation in the renal tubules aggravates tubular necrosis and obstruction with subsequent renal dysfunction. ${ }^{6,7}$ Consequent renal failure from RMinduced myoglobinuria represents about $10-40 \%$ of the total AKI cases. ${ }^{8}$

Although the precise mechanisms implicated in RMrelated AKI are unclear, recent studies have demonstrated that the pathogenesis of AKI encompasses the generation of harmful free radicals and the release of inflammatory mediators. ${ }^{1}$ Furthermore, tubular cell apoptosis is the dominant form of cellular death in the AKI model and is involved in renal injury. In addition to apoptosis, necroptosis is a highly regulated necrosis that depends on phosphorylation of receptor-interacting protein kinase 3 (RIPK3) and strongly contributes to the pathogenesis of AKI. ${ }^{7,9}$ Former in vitro and in vivo investigations found that necroptosis inhibitors as necrostatin-1 or knockdown of RIP3 provoked noteworthy renoprotection in different AKI models. ${ }^{10-12}$

Abundance of experimental evidence was reported that AKI-associated tissue damage can be ameliorated or avoided by natural antioxidants supplementation via suppression of lipid peroxidation and scavenging of excess free radicals. ${ }^{1,3,4,13}$

Lycopene (LYC) is a naturally occurring carotenoid, richly found in tomato and tomato products with potent antioxidant efficacy exceeding other antioxidants such as $\beta$-carotene and $\alpha$-tocopherol. ${ }^{14}$ Its remarkable antioxidant capacity is attributed to numerous double bonds allocated in its chemical structure. ${ }^{15}$ LYC has a strong ability to quench singlet-oxygen and break peroxyl radicals. ${ }^{16}$ Earlier studies have illustrated the antioxidant effect of LYC against renal insult induced by cisplatin, ${ }^{17}$ gentamicin, ${ }^{18}$ methotrexate, ${ }^{15}$ colistin, ${ }^{19}$ and mercuric chloride $^{16}$ toxicities. Also, LYC was shown to markedly relieve the renal inflammation and apoptotic changes in response to diabetic nephropathy and obesity. ${ }^{20,21}$
Selenium, an essential element, is a structural component of antioxidant enzymes as glutathione peroxidases and thioredoxin reductase. ${ }^{22}$ The use of traditional selenium compounds is limited because its safe dose level is narrow, which restricts its application. Therefore, selenium nanoparticles (SeNPs) area more efficient and safe selenium form with high bioavailability and low toxicity. ${ }^{3}$ Preparation of SeNPs is usually done by chemical reduction, using a reducing agent and stabilizer. The use of stabilizers may hamper the biological utilization of SeNPs in addition to their chemical toxicity. ${ }^{23}$ Instead of the chemical method, great attention has been drawn recently to the eco-friendly or green synthesis of SeNPS using biomolecules (plant proteins and polysaccharides) as a capping agent during processing. ${ }^{24-26}$ The eco-friendly approach for SeNPs synthesis is better than other physical and chemical methods due to its low cost, minimum hazard, and high potency. ${ }^{26}$ Recent studies stated that treatment with green synthesized SeNPs had noticeable antioxidant and anti-apoptotic activities. ${ }^{24,27}$

Based on our knowledge, no research exists on the palliative effect of green synthesized SeNPs on the acute renal injury. Hence, this study was performed to elucidate the potential renoprotective properties of LYC-capped SeNPs on renal damage relative to the sole treatment with either sodium selenite or LYC via assessment of oxidative stress, inflammation, apoptotic, and necroptotic signaling pathways.

\section{Materials and Methods \\ Chemicals and Reagents}

Sodium selenite and glycerol were purchased from Sigma Chemical Company, St. Louis, MO, USA. Lycopene was purchased from Puritan's Pride (Ronkonkoma, NY, USA). All other used chemicals were of high analytical grade.

\section{Synthesis of Lycopene Coated-Selenium Nanoparticles (LYC-SeNPs)}

Ten milliliters of $10 \mathrm{mM}$ sodium selenite $\left(\mathrm{Na}_{2} \mathrm{SeO}_{3}\right)$ was mixed with $3.5 \mathrm{mg} / \mathrm{mL}$ LYC (10 $\mathrm{mL})$ under magnetic stirring for $24 \mathrm{~h}$. The obtained solution of LYC-SeNPs was lyophilized by vacuum freeze dryer (Labconco Freezone 4.5 Liter Freeze Dry System, Marshall Scientific, Hampton NH, USA), and the obtained solid powder was utilized in the present study. 


\section{Characterization of the Prepared Nanoparticles (LYC-SeNPs)}

The average diameter, size distribution, and surface charges of the LYC-SeNPs were measured by the Zetasizer Nano ZS particle analyzer (Zetasizer Nano ZS90, Malvern Panalytical, UK). The absorbance of the LYC-SeNPs was measured at wavelengths ranging between 200 and $800 \mathrm{~nm}$ with $1 \mathrm{~nm}$ wavelength intervals using UV-visible spectrophotometer (V-730 UV-visible spectrophotometer, JASCO, Japan). The crystalline size was characterized by X-ray diffraction (XRD; Malvern Panalytical, UK). Furthermore, transmission electron micrographs were recorded using a high-resolution transmission electron microscope (TEM; JEOL Ltd., Japan) equipped with an electron diffraction pattern. The molecular structure of LYC-SeNPs was analyzed using Fourier Transform InfraRed spectroscopy (FTIR; PerkinElmer, USA).

\section{Stability of Nanoparticles in Decreased or Increased $\mathrm{pH}$}

The stability of nanoparticles in decreased or increased $\mathrm{pH}$ was studied by decreasing or increasing the $\mathrm{pH}$ gradually with $0.01 \mathrm{M} \mathrm{HCl}$ or $0.01 \mathrm{M} \mathrm{NaOH}$, respectively, and the appearance of LYC-SeNPs was observed. The starting $\mathrm{pH}$ was approximately 7.0 and the $\mathrm{pH}$ was decreased to 1.0 or increased to 9.0 or until complete dissolution or aggregation of nanoparticles. The $\mathrm{pH}$ was measured with the $\mathrm{pH}$ meter.

\section{Animals and Ethics Statement}

Wistar male albino rats at three months old (150-180 g) were used for this study. They were housed in the animal facility of the Zoology Department, Science of Faculty, Helwan University (Cairo, Egypt) under controlled environmental conditions $\left(24 \pm 2{ }^{\circ} \mathrm{C}\right.$ temperature, $50-60 \%$ relative humidity, and a regular light-dark cycle). Rats were fed on standard rodent-chew diets (Harlan Laboratories Inc., Harlan, Indianapolis, IN; Teklad Diets T.2918) with free access to water. Animal handling and all experimental procedures were performed following the guidelines of the Zoology Department, Faculty of Science, Helwan University (Approval number: HU2019/Z/AER0319-04).

AKI was induced in tested rats via intramuscular (IM) injection of $50 \%$ glycerol $(10 \mathrm{~mL} / \mathrm{kg})$ diluted in saline $(0.9 \% \mathrm{NaCl})$ into the hind limbs with water restriction 24 hours before the injection. ${ }^{28}$

\section{Experimental Protocol}

Rats were allocated into 4 equal groups ( 7 rats per each) as follows:

Group $1(\mathrm{CON})$ : rats served as control and received intramuscular injection with physiological saline $(0.9 \%$ $\mathrm{NaCl})$.

Group 2 (AKI): rats received vehicle for 14 days.

Group $3\left(\mathrm{Na}_{2} \mathrm{SeO}_{3}+\mathrm{AKI}\right)$ : rats were orally administered with $\mathrm{Na}_{2} \mathrm{SeO}_{3}(0.5 \mathrm{mg} / \mathrm{kg})$ for 14 days before being subjected to AKI.

Group 4 (LYC+AKI): rats were orally administered with LYC (10 mg/kg) for 14 days before being subjected to AKI.

Group 5 (LYC-SeNPs+AKI): rats were orally administered with LYC-SeNPs $(0.5 \mathrm{mg} / \mathrm{kg})$ for 14 days before being subjected to AKI.

Twenty-four hours after the last treatment, all animals received an overdose of pentobarbital (100 mg/kg i.p.) and were euthanized by decapitation. Blood samples were collected for biochemical tests. Kidneys were then immediately removed and weighed. The right kidney was used for performing the biochemical and molecular analyses, while the left one was examined for histopathological alterations.

\section{Determination of Kidney Weight}

The relative kidney weight was assessed based on the following mathematical calculation: ${ }^{29}$

\section{Relative kidney weight $=($ Left kidney weight $/$ Body} weight) x 100 .

\section{Preparation of Kidney Homogenate}

A $10 \%(\mathrm{w} / \mathrm{v})$ renal tissue was homogenized in $50 \mathrm{mM}$ Tris- $\mathrm{HCl}(\mathrm{pH}$ 7.4). The homogenate was centrifuged at $4^{\circ} \mathrm{C}$ for $10 \mathrm{~min}$ at $3,000 \times g$. The obtained supernatant was stored at $-80^{\circ} \mathrm{C}$ for biochemical analysis. The renal protein content was measured using bovine serum albumin as a reference protein according to Lowry's method. $^{30}$

\section{Determination of Intensity of Rhabdomyolysis}

Lactate dehydrogenase (LDH) and creatine kinase (CK) were evaluated using kits supplied by Randox/Laboratory, Crumlin, United Kingdom, following the manufacturer's directions. 


\section{Assessment of Renal Function}

\section{Biomarkers}

Levels of urea, creatinine were measured in serum samples by kits (Randox/Laboratory, Crumlin, United Kingdom) based on the manufacturer's information.

\section{Measurement of Kidney Injury}

\section{Molecule-I (Kim-I) and Neutrophil} Gelatinase-Associated Lipocalin (NGAL)

The plasma levels of Kim-1 (R\&D Systems, Catalogue Number: AF3689) and NGAL (MyBioSource, Catalogue Number: MBS260195) were analyzed by ELISA kits following the manufacturer's protocol.

\section{Evaluation of Renal Non-Enzymatic Oxidative Stress Markers}

Peroxidation of lipids was estimated by measured malondialdehyde (MDA). ${ }^{31}$ Further, nitric oxide (NO) content in renal samples was measured by Griess reagent. ${ }^{32}$ Additionally, reduced glutathione (GSH) in renal tissue was evaluated. ${ }^{33}$

\section{Assessment of Kidney Antioxidant Enzymatic Activities}

Superoxide dismutase (SOD) and catalase (CAT) enzymatic activities were measured according to Nishikimi et $\mathrm{al}^{34}$ and Aebi, ${ }^{35}$ respectively. Moreover, glutathione peroxidase (GPx) and glutathione reductase (GR) enzymatic activities were assessed according to Paglia and Valentine $^{36}$ and De Vega et $\mathrm{al}^{37}$ respectively.

\section{Measurement of Inflammatory Biomarkers}

Renal inflammatory reaction was assayed by quantification of tumor necrosis factor- $\alpha$ (TNF- $\alpha$; Catalogue Number: EZRTNFA), interleukin-1 $\beta$ (IL-1 $\beta$; Catalogue Number:
RAB0278), and IL-6 (Catalogue Number: EZRIL6) using kits supplied by Merck Millipore (Toronto, Ontario, Canada) and Sigma-Aldrich (St. Louis, MO, USA), respectively, following the manufacturers' instructions. The levels of measured cytokines were normalized by total protein level.

\section{Assessment of the Renal Apoptotic Markers}

Pro-apoptotic proteins (Cyt $c$, Bax and caspase-3), as well as the anti-apoptotic protein (Bcl-2), were measured by ELISA kit (Cusabio, Wuhan, China) following the manufacturer's procedures. The catalogue Number for Cyt $c$ : CSB-EL006328RA, Bax: CSB-EL002573RA, caspase-3: CSB-E08857r and Bcl-2: CSB-E08854r.

\section{Quantitative Real-Time PCR}

Extraction of total RNA was done from renal tissue by TRIzol reagent, followed by cDNA synthesis using RevertAid $^{\mathrm{TM}}$ H Minus Reverse Transcriptase (Fermentas, Thermo Fisher Scientific Inc., Canada) as mentioned in manufacturer's protocol. qrt-PCR was employed using the QuantiFast SYBR Green RT-PCR kit (Qiagen, Hilden, Germany). All reactions were conducted in duplicate using the ViiA ${ }^{\mathrm{TM}} 7$ System (Thermo Fisher Scientific, CA, USA). The PCR cycling conditions included initial denaturation at $95^{\circ} \mathrm{C}$ for $12 \mathrm{~min}$, followed by 40 cycles of denaturation at $94^{\circ} \mathrm{C}$ for $60 \mathrm{~s}$ and annealing at $58^{\circ} \mathrm{C}$ for $60 \mathrm{~s}$, extension at $72{ }^{\circ} \mathrm{C}$ for $90 \mathrm{~s}$, then held for a final extension at $72^{\circ} \mathrm{C}$ for $10 \mathrm{~min}$. The relative gene expression was determined between the different groups using the ${ }^{\Delta \Delta} \mathrm{Ct}$ method. ${ }^{38}$ Glyceraldehyde-3-phosphate dehydrogenase (Gapdh) was used as a housekeeping gene. The primer sequences (Jena Bioscience [Jena, Germany]) for estimation of Nef2, Hmox-1, Nos2, and Ripk3 gene expressions are listed in Table $1 .{ }^{39}$

Table I Primer Sequences of Genes Analyzed in Real Time-PCR

\begin{tabular}{|l|l|l|l|}
\hline Name & Accession Number & Sense $\left(\mathbf{5}^{\prime} \mathbf{-} \mathbf{3}^{\prime} \mathbf{)}\right.$ & Antisense $\mathbf{( 5 ' - \mathbf { 3 } ^ { \prime } )}$ \\
\hline Gapdh & NM_017008.4 & AGTGCCAGCCTCGTCTCATA & TCCCGTTGATGACCAGCTTC \\
Nfe2I2 & NM_031789.2 & CAGCATGATGGACTTGGAATTG & GCAAGCGACTCATGGTCATC \\
Hmoxl & NM_012580.2 & TTAAGCTGGTGATGGCCTCC & GTGGGGCATAGACTGGGTTC \\
Ripk3 & NM_139342.1 & GAGACCCAAGCTGAGCCTAA & ACGTCTACAACGTCTACGGC \\
Nos2 & NM_0126II.3 & GGTGAGGGGACTGGACTTTTAG & TTGTTGGGCTGGGAATAGCA \\
\hline
\end{tabular}

Abbreviations: Gapdh, Glyceraldehyde 3-phosphate dehydrogenase; Nfe2I2, Nuclear factor-erythroid 2-related factor 2; HmoxI, Heme oxygenase I; Ripk3, Receptor Interacting Serine/Threonine Kinase 3; Nos2, Nitric oxide synthase 2. 


\section{Light Microscopy and Histopathological Screening}

The left kidneys from all studied groups were fixed in $10 \%$ neutral buffered formalin for $24 \mathrm{~h}$. Tissue samples were dehydrated, embedded in paraffin, and finally sectioned at $8 \mu \mathrm{m}$ thick sections. Renal specimens were stained with hematoxylin and eosin (H\&E) and examined under a Nikon Eclipse E200-LED (Tokyo, Japan) microscope at $400 \times$ magnification.

\section{Statistical Analysis}

Obtained data were analyzed by one-way analysis of variance (ANOVA) and Duncan's post-hoc multiple tests to assess the differences between the groups. The results were displayed as mean \pm standard error of the mean (SEM). The level of statistical significance was set at $P$-values lower than $0.05(P<0.05)$.

\section{Results}

\section{Physical and Chemical Characterization of the Prepared LYC-SeNPs}

When adding LYC to $\mathrm{Na}_{2} \mathrm{SeO}_{3}$, the color changed from colorless to red color (Figure 1A). SeNPs were characterized with an average diameter of $129.3 \mathrm{~nm}$ (Figure 1B) and a mean zeta potential of $-25.3 \mathrm{mV}$ (Figure 1C). The UV-visible spectroscopic analysis of the LYC-SeNPs revealed that the maximum absorption $\left(\lambda_{\max }\right)$ was recorded at $353 \mathrm{~nm}$ after one day of preparation (data not shown). Furthermore, the XRD pattern result showed a broader pattern without any definite Braggs peaks. The obtained results indicate that LYCSeNPs are not crystalline; rather they are more amorphous (Figure 1D). The prepared LYC-SeNPs-calculated crystalline size is $<102 \mathrm{~nm}$ using Scherrer's equation. This amorphous character in agreement with the earlier studies carried out with Withania somnifera ${ }^{39}$ and Bacillus sp. ${ }^{40}$ Moreover, TEM is employed to analyze the shape of the LYC-SeNPs biosynthesized LYC. TEM image of LYC-SeNPs revealed spherical particles within the diameter $<130 \mathrm{~nm}$. These particles are well distributed with no aggregation (Figure 1E). The FT-IR analysis result of synthesized LYC-SeNPs is depicted in Figure 1F. A broad peak observed at $3306.17 \mathrm{~cm}^{-1}$ corresponds to $\mathrm{O}-\mathrm{H}$ stretch alcohols and phenols. The absorption peak at $2129.66 \mathrm{~cm}^{-1}$ corresponds to $\mathrm{C}-\mathrm{H}$ stretch alkynes. The band at $1635.65 \mathrm{~cm}^{-1}$ is due to $\mathrm{C}-$ $\mathrm{O}$ asymmetric stretch carbon compounds. The absorption peak at $1039.05 \mathrm{~cm}^{-1}$ is attributed to the $\mathrm{C}-\mathrm{N}$ stretching of the amines. $\mathrm{C}-\mathrm{X}$ stretching in alkyl halides causes a band at $602.98 \mathrm{~cm}^{-1}$. These results reveal the presence of various functional groups that may be responsible for both reduction and stabilization of the SeNPs.

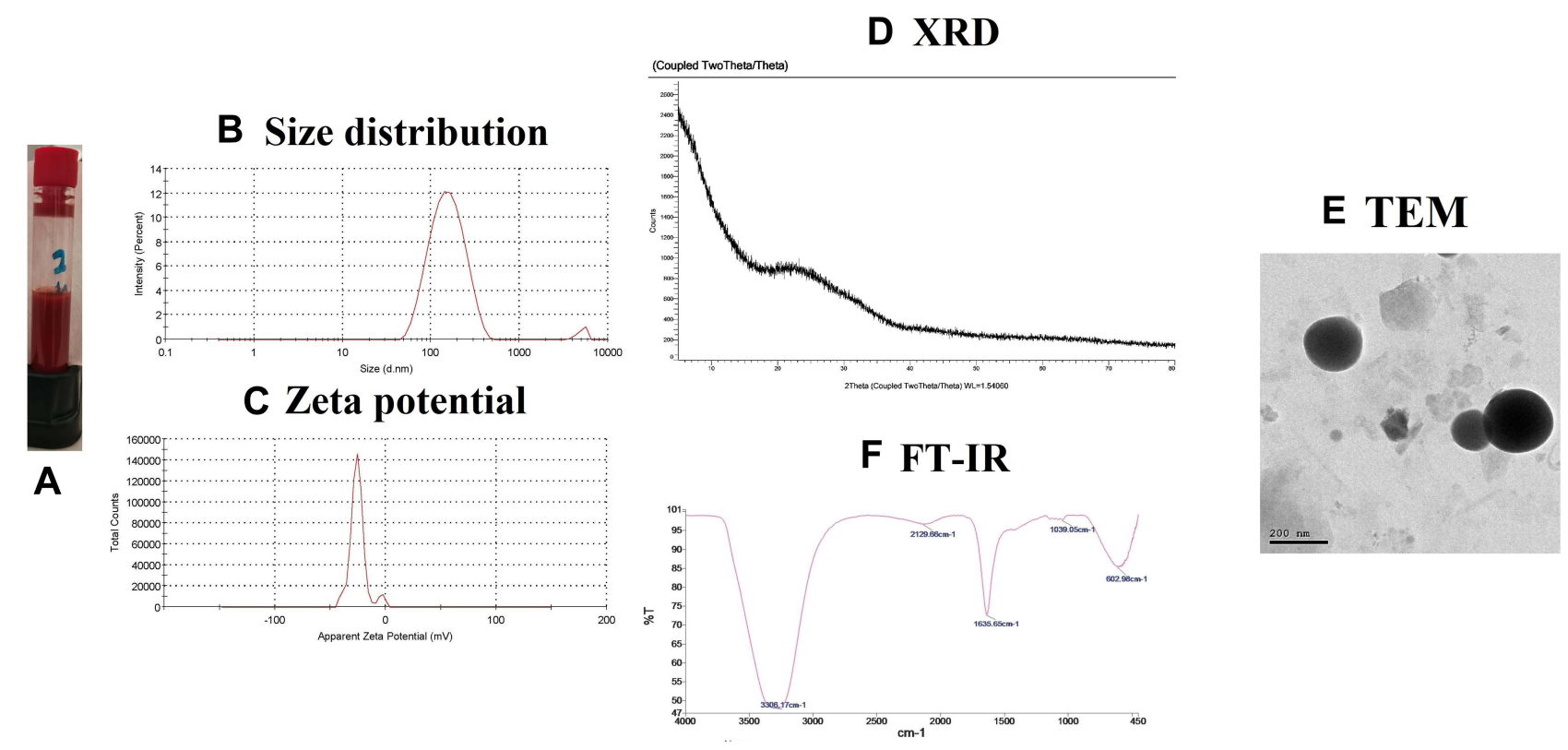

Figure I Characterization of lycopene-coated selenium nanoparticles (LYC-SeNPs). (A) Color of LYC-SeNPs at 24 h. (B) Hydrodynamic diameter of LYC-SeNPs by Zetasizer. (C) Surface charge of LYC-SeNPs by Zeta potential. (D) XRD spectra of LYC-SeNPs. (E) Morphological shape of LYC-SeNPs as observed by TEM. (F) FT-IR spectra of LYC-SeNPs. 
The LYC-SeNPs nanoparticles were stable in decreased $\mathrm{pH}$ till 1.0. However, the nanoparticles aggregated and/or precipitated as $\mathrm{pH}$ was increased 11.3, but when the $\mathrm{pH}$ was decreased again, the LYC-SeNPs were resolved and the color changed from colorless to red color.

\section{LYC-Capped SeNPs Improved}

\section{RM-Related Biomarkers in the AKI Model}

The sequences of $\mathrm{LYC}, \mathrm{Na}_{2} \mathrm{SeO}_{3}$, and LYC-SeNPs supplementation on creatine kinase and LDH in glycerol-injected rats are shown in Figure 2. Marked increases $(P<0.05)$ in RM indicators were observed in the AKI group related to the control group. In contrast, when glycerol-injected rats were co-administrated with $\mathrm{LYC}$ or $\mathrm{Na}_{2} \mathrm{SeO}_{3}$, substantial declines $(P<0.05)$ in RM parameters were detected compared to the AKI group. Interestingly, the co-treatment with LYC-SeNPs resulted in marked declines $(P>0.05)$ in LDH level compared to AKI and LYC administered group but not different from $\mathrm{Na}_{2} \mathrm{SeO}_{3}$ group. Creatine kinase level displayed a pronounced decrease $(P<0.05)$ in the LYC-SeNPs group relative to the AKI and the treated groups with $\mathrm{LYC}$ and $\mathrm{Na}_{2} \mathrm{SeO}_{3}$. These results suggested that administration of LYC-SeNPs exerted better protection on muscle fiber damage than the effect of either LYC or selenium alone.

\section{Effect of LYC-Loaded SeNPs}

\section{Administration on Kidney Weight and}

\section{Function Markers}

The mitigating effect of LYC-SeNPs on renal damage in glycerol-injected rats is illustrated in Figure 3. Significant increments $(P<0.05)$ were detected in both kidney weight and relative kidney weight in the glycerol group relative to the control one, while their levels showed notable decreases $(P<0.05)$ in the treated groups compared to the AKI group. No significant changes $(P>0.05)$ were noticed upon comparing the treated groups with LYC, $\mathrm{Na} 2 \mathrm{SeO} 3$, or LYC-SeNPs in terms of kidney weight or relative kidney weight. Regarding the serum kidney function markers (urea and creatinine), markedly elevated levels $(P<0.05)$ were recorded in the AKI group with respect to the control group (Figure 3). However, their levels exhibited marked decreases $(P<0.05)$ in groups that received $\mathrm{Na}_{2} \mathrm{SeO}_{3}, \mathrm{LYC}$, or LYC-SeNPs compared to the AKI group. It is noteworthy that LYC-SeNPs coadministration evoked significant declines $(P<0.05)$ in creatinine levels compared with the sole LYC or $\mathrm{Na}_{2}$ $\mathrm{SeO}_{3}$ treatment.

Levels of NGAL and Kim-1 showed significant elevations $(P<0.05)$ following glycerol injection (Figure 3$)$. Meanwhile, co-treatment with $\mathrm{Na}_{2} \mathrm{SeO}_{3}$, LYC, or LYCSeNPs significantly reduced the concentrations of NGAL and Kim-1 compared to the untreated AKI group. Furthermore, the group co-treated with LYC-SeNPs had the lowest levels $(P<0.05)$ of both NGAL and Kim-1 compared to the treated groups.

\section{Augmentation of Renal Antioxidant Response After LYC-SeNPs Supplementation}

The impact of different treatments on the renal tissue's redox status is represented in Figures 4 and 5. Marked oxidant/antioxidant imbalance was observed in the AKI group, evidenced by significant elevations $(P<0.05)$ in MDA and NO levels paralleled with marked depletion
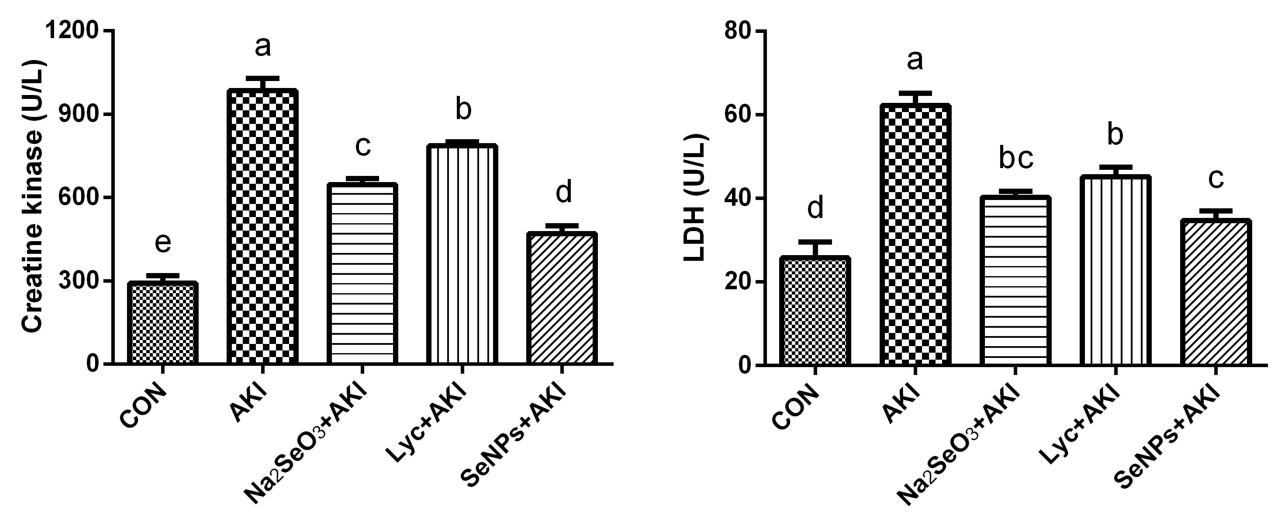

Figure 2 The effect of lycopene coated selenium nanoparticles (LYC-SeNPs) on the rhabdomyolysis related parameters in glycerol-induced AKI model in rats. Data are expressed as mean \pm SEM, $n=7$. The statistical difference between groups was estimated using Duncan's post-hoc test at $P<0.05$. Bars that do not share same letters (superscripts) are significantly different from each other $(p<0.05)$. 

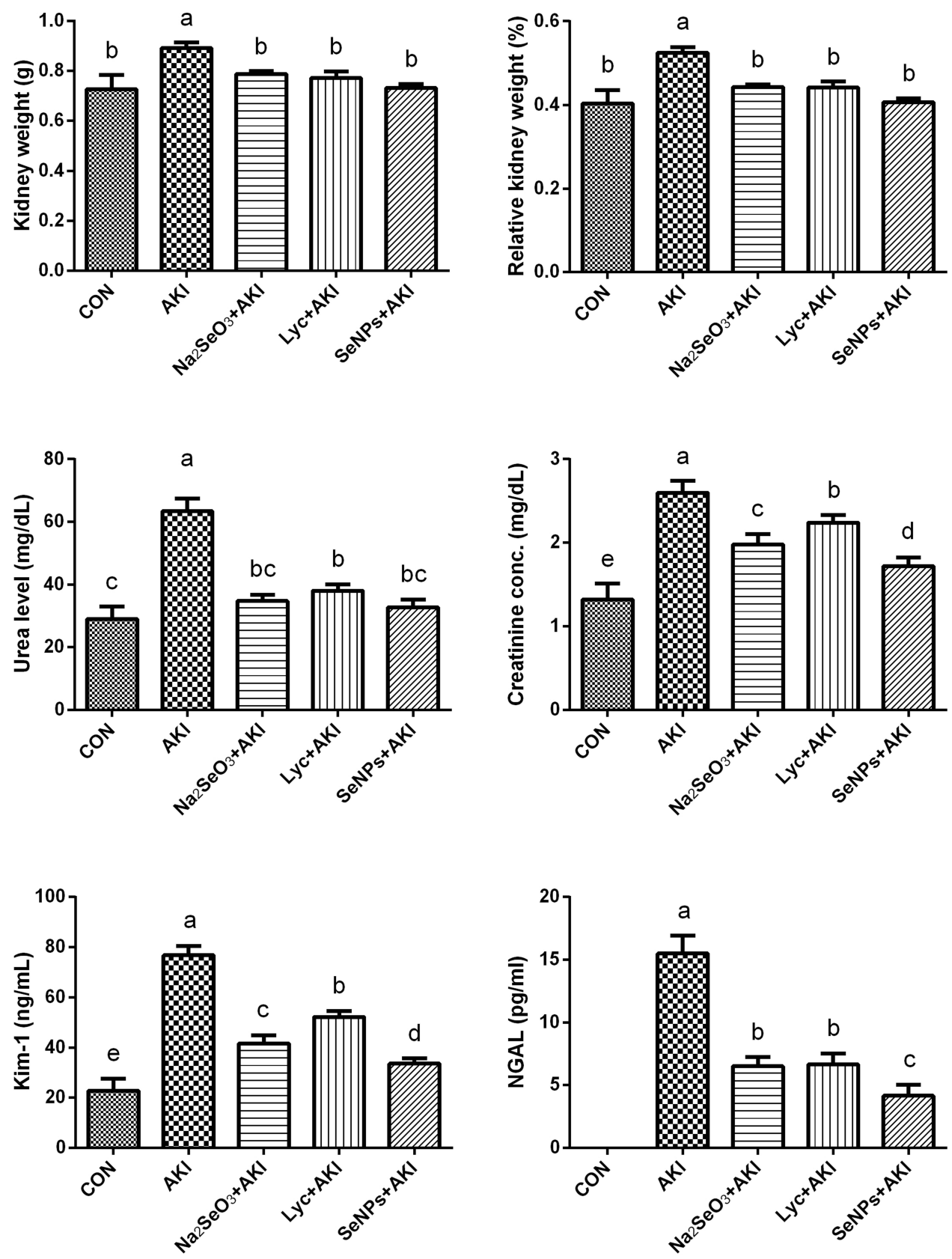

Figure 3 The effect of lycopene coated selenium nanoparticles (LYC-SeNPs) on kidney weight and renal function markers in glycerol-induced acute kidney injury. Data are expressed as mean \pm SEM, $n=7$. The statistical difference between groups was estimated using Duncan's post-hoc test at $P<0.05$. Bars that do not share same letters (superscripts) are significantly different from each other $(p<0.05)$.

$(P<0.05)$ in GSH level with respect to the control group. Moreover, prominent suppressions $(P<0.05)$ in the antioxidant enzymatic activities of SOD, CAT, GPx, and GR after glycerol injection were observed. In contrast, co-treatment of the AKI group with $\mathrm{Na}_{2} \mathrm{SeO}_{3}$, LYC, or LYC-SeNPs improved the redox status in the renal tissue via enhancement $(P<0.05)$ of the antioxidant defense members and GSH content $(P<0.05)$ accompanied by diminished $(P<0.05)$ MDA and NO levels when compared with the AKI untreated group. Co-administration with LYC-SeNPs resulted in significant GSH level increases and GPx accompanied by a decline in MDA level with respect to the other treated groups. Remarkably, LYC-SeNPs supplementation reversed the oxidative stress in renal tissue as indicated by the restoration of antioxidant enzymatic activities (SOD, CAT, and GR) and GSH level near to their corresponding values in the control group. 

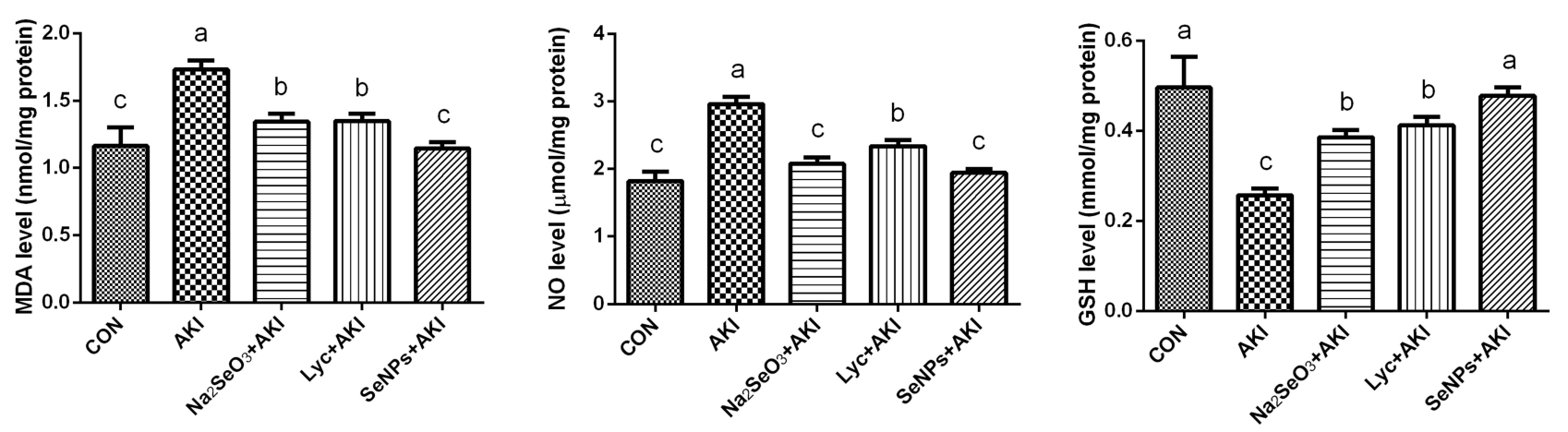

Figure 4 The effect of lycopene coated selenium nanoparticles (LYC-SeNPs) on non-enzymatic antioxidant parameters [malondialdehyde (MDA), nitric oxide (NO), and glutathione (GSH)] levels in glycerol-induced AKI in rats. Data are expressed as mean \pm SEM, $n=7$. The statistical difference between groups was estimated using Duncan's post-hoc test at $P<0.05$. Bars that do not share same letters (superscripts) are significantly different from each other ( $P<0.05)$.
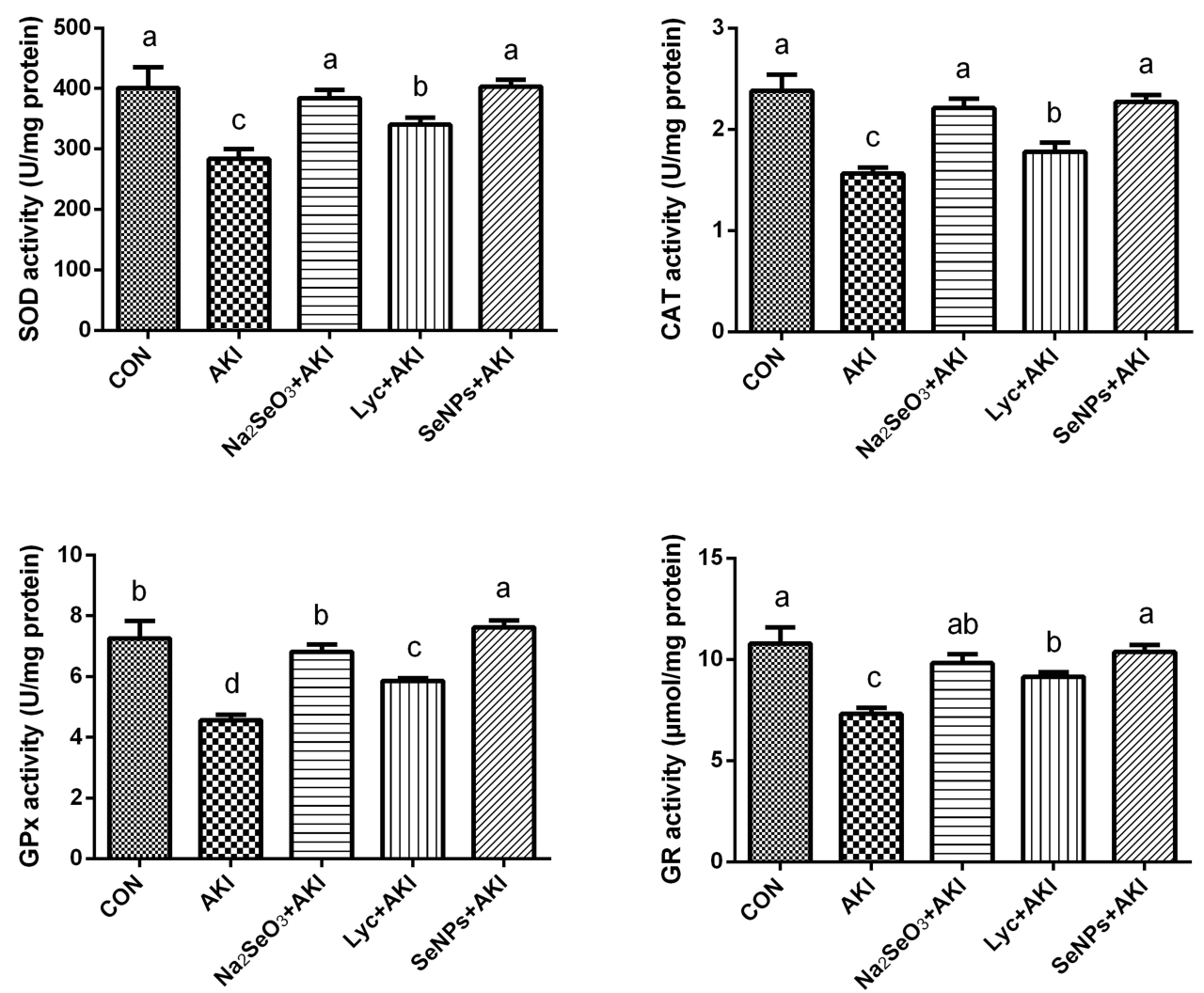

Figure 5 The effect of lycopene coated selenium nanoparticles (LYC-SeNPs) on antioxidant enzymatic activities in glycerol-induced acute kidney injury. Data are expressed as mean \pm SEM, $n=7$. The statistical difference between the control and glycerol injected groups was estimated using Duncan's post-hoc test at $P<0.05$. Bars that do not share same letters (superscripts) are significantly different from each other $(p<0.05)$.

Abbreviations: GPx, glutathione peroxidase, GR, glutathione reductase; SOD, superoxide dismutase, CAT, catalase.

On the molecular basis, marked downregulations $(P<$ $0.05)$ were detected in mRNA expression of nuclear factor, erythroid derived 2, like 2 (Nfe212), and heme oxygenase 1 (Hmoxl) in the glycerol-induced AKI with respect to the control groups. In contrast, $\mathrm{Na}_{2} \mathrm{SeO}_{3}$ or LYC-treated rats had higher expression levels $(P<0.05)$ of these antioxidant-related genes than rats with AKI. Lycopene capped SeNPs administration upregulated their expressions markedly $(P<0.05)$ with respect to the LYC or $\mathrm{Na}_{2}$ $\mathrm{SeO}_{3}$ treated groups except for the non-significant difference in Hmox-1 expression level in $\mathrm{Na}_{2} \mathrm{SeO}_{3}$ treated group (Figure 6). 

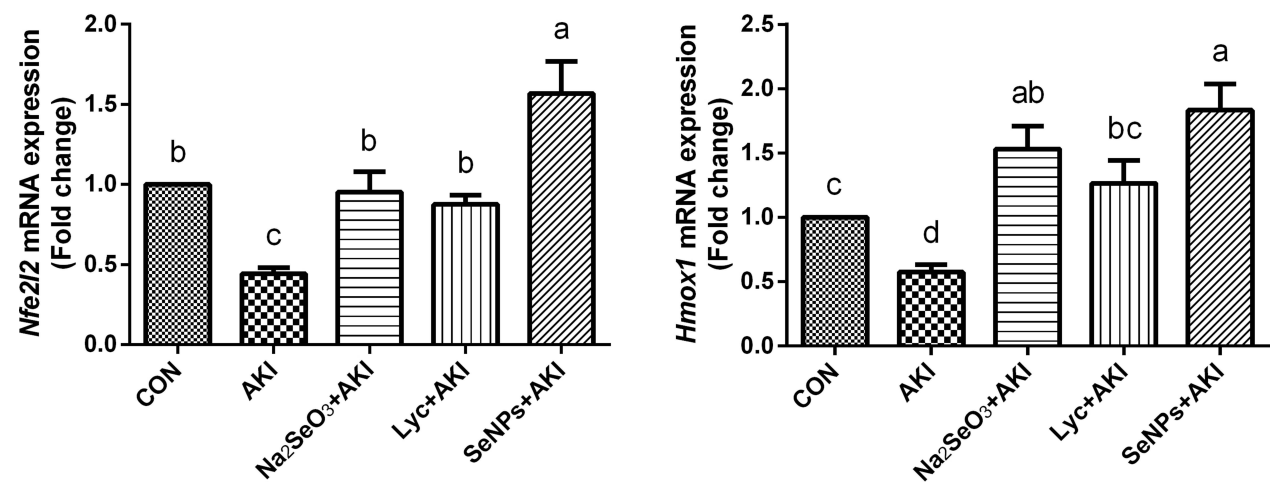

Figure 6 The effect of lycopene coated selenium nanoparticles (LYC-SeNPs) on mRNA expression of Nfe2/2 and Hmox-I in glycerol-induced AKI in rats. Data are expressed as mean $\pm S E M, n=7$. The obtained results were demonstrated as the mean \pm SEM of triplicate experiments and were referenced to Gapdh and represented as a fold change ( $\log 2$ scale), with respect to mRNA levels in the control group. The statistical difference between groups was estimated using Duncan's post-hoc test at $P<$ 0.05 . Bars that do not share same letters (superscripts) are significantly different from each other $(p<0.05)$.

\section{LYC-Coated SeNPs Alleviated Glycerol-Induced Renal Inflammation in the AKI Model}

Compared to the renal tissue in the control group, significant increases $(P<0.05)$ were observed in the IL-1 $\beta$, IL-6, and TNF- $\alpha$ levels associated with upregulated Nos 2 expression level $(P<0.05)$ in the kidney tissue of glycerolinjected rats. Surprisingly, this inflammation situation in renal tissue was attenuated $(P<0.05)$ by the $\mathrm{Na}_{2} \mathrm{SeO}_{3}$, LYC, or LYC-SeNPs administration, as shown by the reversal in all parameters mentioned above. Selenium nanoparticles loaded on LYC differed significantly $(P<$ 0.05 ) from the LYC-treated group in terms of IL-1 $\beta$ and TNF- $\alpha$ levels (Figure 7).

\section{Amelioration of LYC-Conjugated SeNPs on Apoptosis and Necroptosis in Glycerol-Induced AKI}

Glycerol-mediated AKI is associated with significant induction of apoptotic and necroptotic pathways. As shown in Figure 8, significant elevations $(P<0.05)$ in proapoptotic proteins (caspase-3, Bax, and cyt-c) levels accompanied by a marked decline $(P<0.05)$ in antiapoptotic protein (Bcl-2) were detected in kidneys with glycerol-induced tissue damage. Further, marked upregulation in mRNA expression of Ripk3, a necroptotic marker, in glycerol injected rats compared to the control group. In contrast, these parameters were markedly reduced $(P<$ $0.05)$ when glycerol-injected rats were administered with $\mathrm{Na}_{2} \mathrm{SeO}_{3}$, LYC, or LYC-SeNPs compared to the AKI group. Notably, the application of LYC capped SeNPs treatment in the AKI group and reversed significantly
$(P<0.05)$ the apoptotic damage in caspase-3, cyt-c, and Bcl-2 compared to rats administered with LYC or $\mathrm{Na}_{2}$ $\mathrm{SeO}_{3}$ alone. These data suggested that when LYC coated SeNPs formulation was used, it provided better protection against AKI-induced renal damage than either agent alone.

\section{Effect of LYC-Loaded SeNPs Administration on Renal Histopathological Changes}

Microscopic screening of stained renal sections from the control group showed normal tissue architecture of the renal cortex and medulla (Figure 9). In contrast, sections from the glycerol-treated group revealed extensive tissue damage demonstrated by tubular dilation, vacuolation, necrosis, and debris accumulation in the tubular lumina. However, renal sections from rats treated with $\mathrm{Na}_{2} \mathrm{SeO}_{3}$ or LYC showed some improvement of the renal histological changes. Interestingly, LYC-SeNPs supplementation contributed to a notable improvement in the renal tissue alteration.

\section{Discussion}

Selenium nanoparticles have attracted extensive interest in the fields of nanomedicine owing to their unique biological activities. In particular, biogenic SeNPs synthesized by green nanotechnology represent promising and safe selenium supplements with low toxicity, high bioavailability, low cost, and eco-friendly processing. ${ }^{41}$ In this regard, our study herein illustrated the potential of SeNPs synthesized using LYC as a therapeutic approach to alleviate AKIrelated tissue damage in terms of oxidative stress, inflammation, apoptosis, and necroptosis. 

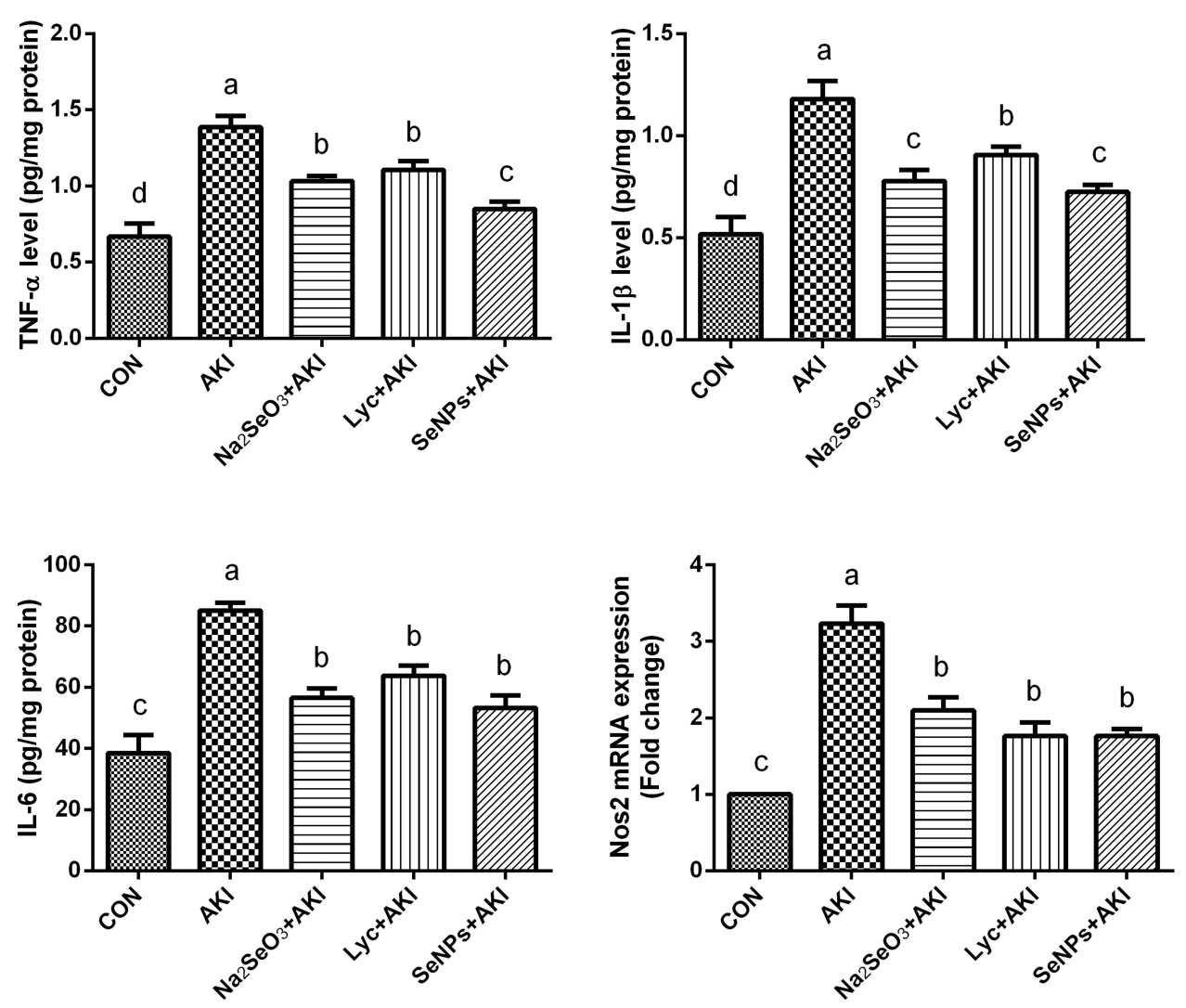

Figure 7 The effect of lycopene coated selenium nanoparticles (LYC-SeNPs) on the levels of inflammatory biomarkers (TNF- $\alpha$, IL-I $\beta$ and IL-6) and Nos2 mRNA expression in glycerol-induced AKI in rats. For ELISA results, data are expressed as mean \pm SEM, $n=7$. The statistical difference between the control and glycerol injected groups was estimated using Duncan's post-hoc test at $P<0.05$. Bars that do not share same letters (superscripts) are significantly different from each other ( $P<0.05$ ). For $q R T-P C R$ findings, the obtained results were demonstrated as the mean \pm SEM of triplicate experiments and were referenced to Gapdh and represented as a fold change (log2 scale), with respect to mRNA levels in the control group.

Glycerol injection is commonly used to induce AKI model due to skeletal muscle degeneration and leakage of its content into the circulation, which induces a myoglobinuric state similar to clinical RM. Consistent with previous studies, our results revealed marked increases in muscle damage markers (serum creatine kinase and LDH) in the glycerol-injected group compared to the control group. ${ }^{1,3,5}$ The released products have been reported to undergo glomerular filtration causing intratubular obstruction, renal vasoconstriction, inflammatory response, and production of reactive oxygen species leading to AKI. ${ }^{3,4}$ Renal impairment was evident in the AKI group as witnessed by prominent elevations in serum urea and creatinine levels associated with elevated relative kidney weight as previously reported. ${ }^{5,7}$ In support, renal tissue histopathological alterations revealed tubular ischemia, vacuolation, and subsequent tubular necrosis and glomerular injury. Additionally, KIM-1, as a specific marker of renal injury located in damaged renal epithelial cells in the proximal tubule, displayed a marked increase in the
AKI group, which is consistent with former studies., ${ }^{7,42}$ NGAL, stress protein, belongs to the lipocalin superfamily and is considered an early diagnostic marker for AKI. ${ }^{43} \mathrm{In}$ agreement with Sharawy et $\mathrm{al}^{43}$ a significant increment was found in its level in the glycerol-treated group that ensures renal damage.

Notably, LYC or $\mathrm{Na}_{2} \mathrm{SeO}_{3}$ treatment demonstrated marked alleviation in biomarkers related to RM and renal damage and renal pathological alterations supporting their capacities to improve kidney function and safeguard glycerol-induced damage. Earlier investigations indicated LYC treatment's renoprotective effect against nephrotoxic agents. ${ }^{16-18}$ Additionally, Tsitsimpikou et $\mathrm{al}^{44}$ found that LYC efficiently restored the levels of LDH and creatine kinase levels to almost normal levels in exercise-induced myocardial damage. The carotenoid LYC's renoprotection may be attributed to distinguished antioxidant and free radical scavenging capacity and its cytoprotective efficacy against proteins, lipids, and DNA oxidation. ${ }^{19}$ Similarly, sodium selenite co-treatment improved the renal functions 

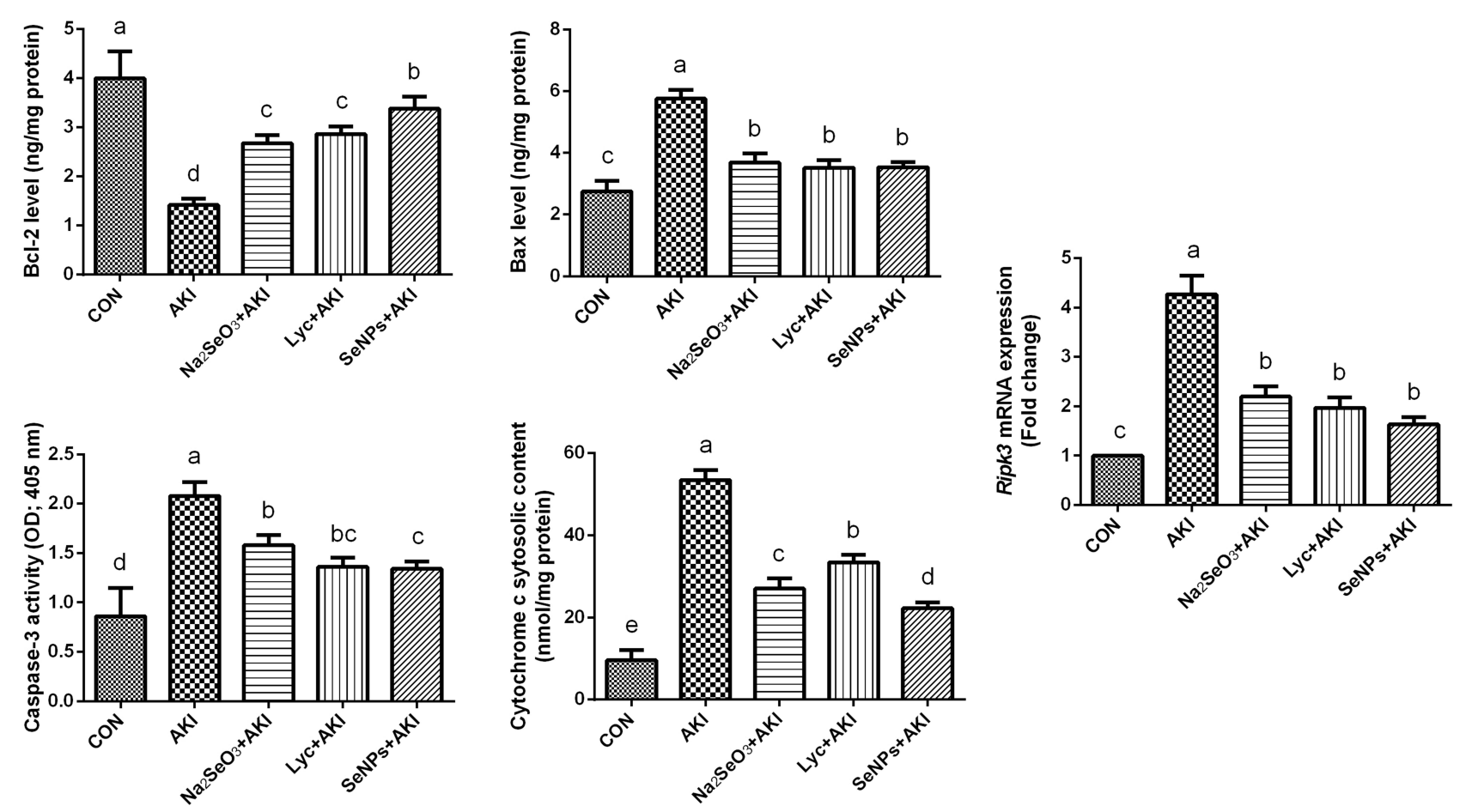

Figure 8 The effect of lycopene coated selenium nanoparticles (LYC-SeNPs) on the levels of apoptotic and necroptotic related markers in glycerol-induced AKI in rats. For ELISA results, data are expressed as mean \pm SEM, $n=7$. The statistical difference between groups was estimated using Duncan's post-hoc test at $P<0.05$. Bars that do not share same letters (superscripts) are significantly different from each other $(p<0.05)$. For qRT-PCR findings, the obtained results were demonstrated as the mean \pm SEM of triplicate experiments and were referenced to Gapdh and represented as a fold change (log2 scale), with respect to mRNA levels in the control group.

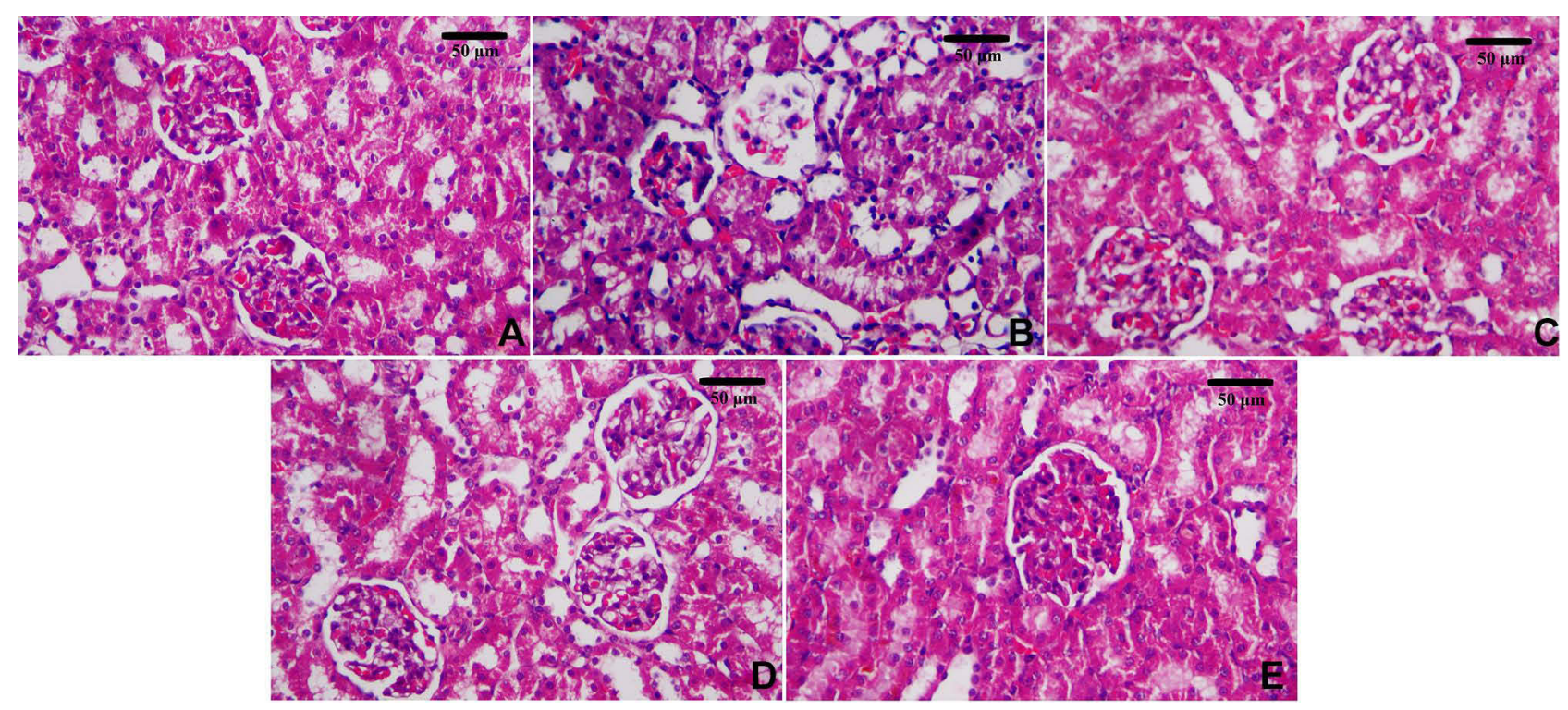

Figure 9 Histopathological alterations in the renal tissue following glycerol injection and different treatments. (A) Control, (B) AKI, (C) $\mathrm{Na}{ }_{2} \mathrm{SeO}{ }_{3}+\mathrm{AKI},(\mathbf{D}) \mathrm{LYC}+\mathrm{AKI}$, and (E) LYC-SeNPs+AKI. Hematoxylin and eosin (H\&E), scale bar $=50 \mu \mathrm{m}$.

and relieved the histological alterations induced by glycerol. These findings are attributed to its potent antioxidant capacity and are consistent with previous results. ${ }^{3,45,46}$ Interestingly, green synthesized SeNPs using LYC provided more significant renoprotection than using sodium selenite or LYC alone. Krishnan et $\mathrm{al}^{24}$ reported that SeNPs loaded with Spermacoce hispida exhibited many nephroprotective and hepatoprotective effects against acetaminophen toxicity compared to the sole treatment with SeNPs in rats. Similarly, Ramamurthy et $\mathrm{al}^{26}$ stated that 
green synthesized SeNPs using fenugreek seed extract notably inhibited the cell growth in human breast cancer cells in dose-dependent manner. Further, LYC-rich nanoemulsions recorded the best protection of cardiomyoblast against doxorubicin toxicity via its excellent cytoprotective properties. ${ }^{47}$

Multiple mechanisms are contributing to the pathogenesis of glycerol-induced AKI. The muscle damage induced by glycerol results in the release of products such as myoglobin and heme derivates into the bloodstream. These products have a catalytic iron metal center, which initiates free radicals production with consequent lipid peroxidation and oxidative stress in the proximal renal tubules. ${ }^{1,42}$ In the present study, renal tissue of the AKI model exhibited marked elevations in MDA and NO levels paralleled with a decline in GSH and suppression of SOD, CAT, GPx, and GR enzymatic activities. These findings agree with former authors. ${ }^{4,42}$ Supporting previous reports, the current study revealed notable downregulations in mRNA expressions of both Nrf2 and HO-1 in renal tissue of the AKI group. ${ }^{4,48} \mathrm{Nrf} / \mathrm{HO}-1$ signaling pathway is reported to contribute to the oxidative injury in rhabdomyolysis-induced AKI. ${ }^{4,48} \mathrm{Nrf} 2$ is a master transcriptional factor regulating a battery of genes encoding antioxidant proteins, like HO- $1 .{ }^{49} \mathrm{HO}-1$ can convert heme to biliverdin and produce carbon monoxide with a cytoprotective role. ${ }^{1,49}$ Because of its importance in the catabolic process of heme protein, it is suggested that HO-1 is included in the catabolism of myoglobin in damaged renal tissues. ${ }^{48}$

LYC is considered the most effective antioxidant carotenoid, owing to single oxygen radical and other reactive species' scavenging ability. During the quenching of ${ }^{1} \mathrm{O}_{2}$, the LYC converts to a high energy-rich molecule, capable of trapping other ROS, like $\mathrm{OH}, \mathrm{NO}_{2}$, or peroxynitrite. However, further trapping to other radicals leads to the oxidative breakdown of LYC. ${ }^{16,19}$ In our study, LYC recovered the oxidant/antioxidant imbalance in glycerolinduced renal injury markedly. These results are consistent with former reports. ${ }^{16,17,50}$ Furthermore, the molecular analysis confirmed the antioxidant potential of LYC by notable stimulation for the Nrf2/HO-1 signaling pathway, which is concomitant with Dai et al. ${ }^{19}$

Owing to its vital incorporation into seleniumdependent enzymes as GPx and thioredoxin reductases, selenium can adequately handle the free radicals and maintain the redox balance inside the cells. ${ }^{3,46}$ Our findings revealed marked improvement in the cellular antioxidant capacity in renal tissue of sodium selenite-treated group, which is in agreement with earlier authors. ${ }^{46}$ Using LYC for the synthesis of SeNPs in our study provided distinguished antioxidant activities compared to the cotreatment with LYC or sodium selenite. Zhang et $\mathrm{al}^{25}$ stated that the SeNPs capped with Lycium barbarum polysaccharides had powerful antioxidant ability, and they have a protective effect against $\mathrm{H}_{2} \mathrm{O}_{2}$-induced cell death in the PC-12 cell line. It is assumed that the beneficial effect of LYC-SeNPs is endorsed for the synergistic effects that can be generated between plant polysaccharides and SeNPs in addition to the increased bioavailability and bioactivity. ${ }^{27}$

Activation of pro-inflammatory pathways and renal inflammation is a typical pathophysiological feature of rhabdomyolysis-induced AKI, which may exacerbate tubular epithelium damage and fibrosis. ${ }^{3,6,7}$ In our study, significant increments were detected in the levels of IL- $1 \beta$, TNF- $\alpha$ and IL-6, as well as the fold change of Nos 2 in the glycerolinjected group. Such a strong inflammatory response in the AKI model may be attributed to excess ROS production and activation of nuclear factor kappa-B (NF- $\mathrm{KB}$ ), which controls the expression of iNOS and pro-inflammatory cytokines. ${ }^{2}$ Additionally, iNOS triggers the generation of reactive nitrogen species that also interact with superoxide anions to produce peroxynitrite radical and enhance the production of pro-inflammatory cytokines. ${ }^{43}$

Marked lessening of the renal inflammation was observed in LYC or sodium selenite co-treated rats as indicated by lowered IL-1 $\beta$, IL- 6 , and TNF- $\alpha$ and downregulated iNOS expression, consistent with the findings of earlier studies. ${ }^{20,21}$ The anti-inflammatory action of LYC refers to the inhibition of nuclear factor kappa $\mathrm{B}$ and the production of proinflammatory mediators. Likewise, earlier reports stated that elenium exerts its anti-inflammatory activity via suppressingNF- $\kappa \mathrm{B},{ }^{3}$ iNOS, and COX-2. ${ }^{49}$ Nanoformulation of selenium based on LYC achieved a marked reduction in TNF- $\alpha$ level than the individual LYC or selenium did, indicating the optimized bioavailability of SeNPs. Quagliariello et $\mathrm{al}^{47}$ stated that lycopene-rich nanoemulsion potentially lessened the inflammatory cytokines and NO in cardiomyocytes exposed to doxorubicin. Also, SeNPs coated with Ulva lactuca polysaccharide markedly diminished the inflammation in acute colitis. ${ }^{51}$

In addition to the discussed pivotal role of oxidative stress and inflammation in renal injury, apoptosis and necroptosis of renal tubular epithelial cells are also crucial in glycerolinduced AKI's pathophysiology. In our study, noteworthy increases were recorded in renal Bax, caspase-3, and Cyt-c 
levels associated with a marked decline in Bcl2 in the glycerol-injected group indicating a pronounced shift towards the direction of apoptosis. Myoglobin-induced oxidative stress alters the mitochondrial permeability with the subsequent release of cytochrome-c and caspase activation. ${ }^{52}$ These findings were in line with former studies., ${ }^{3,5}$ Necroptosis, a type of regulated necrosis, is a caspaseindependent death pathway involved in glycerol induced AKI. ${ }^{52}$ Also, renal injury induced by glycerol was accompanied by a marked upregulation in Ripk3 expression. It was formerly documented that TNF- $\alpha$ has a crucial role in triggering the necroptotic signaling pathway via interaction with TNF receptor 1 (TNFR1) and phosphorylation of RIPK3 by RIPK1. Phosphorylated RIPK1 and RIPK3 activate mixed lineage kinase domain-like pseudokinase (MLKL), which also control necroptosis with RIPK. ${ }^{5}$ This result came in line with previous authors. ${ }^{5,7,52}$

Co-treatment with LYC evoked marked anti-apoptotic and anti-necroptotic activities in the glycerol-injected group, which is in agreement with previous studies. ${ }^{53,54}$ The significant cytoprotective effect of LYC in renal tissue may refer to its antioxidant potential, activation of the Nrf2 pathway paralleled with suppression of the NF- $\mathrm{kB}$, and caspase/Bcl2 apoptotic pathways. ${ }^{19}$ It was reported that administration of necrostatin-1, a RIPK1 inhibitor, reduced creatinine plasma levels and tubular necrosis. ${ }^{55}$ Accordingly, the anti-necroptotic effect of LYC or selenium is assumed to be related to their inhibitory action on Ripk3. In support of former results, ${ }^{27,45}$ LYC-SeNPs exerted an anti-apoptotic effect compared to LYC or selenium only due to suppression of caspases.

\section{Conclusion}

Taken together, biogenic SeNPs synthesized by LYC exhibited considerable nephroprotective activity against AKI-caused tissue damage in rat models. The possible mechanisms of LYC-SeNPs effects may be attributed to free radical scavenging ability with the enhancement of the endogenous antioxidant system, attenuating the renal inflammation, anti-apoptotic and anti-necroptotic activities. These findings suggested that the synthesis of SeNPs by LYC is a promising selenium supplement, which is a novel therapeutic strategy in the amelioration of AKI-related tissue damage.

\section{Funding}

This work was supported by Taif University Researchers Supporting Program (Project number: TURSP-2020/151), Taif University, Saudi Arabia.

\section{Disclosure}

The authors declare no conflicts of interest in this work.

\section{References}

1. Yin M, Jiang N, Guo L, et al. Oleuropein suppresses oxidative, inflammatory, and apoptotic responses following glycerol-induced acute kidney injury in rats. Life Sci. 2019;232:116634. doi:10.1016/ j.1fs.2019.116634

2. Makris K, Spanou L. Acute kidney injury: definition, pathophysiology and clinical phenotypes. Clin Biochem Rev. 2016;37(2):85.

3. AlBasher G, Alfarraj S, Alarifi S, et al. Nephroprotective role of selenium nanoparticles against glycerol-induced acute kidney injury in rats. Biol Trace Elem Res. 2020;194(2):444-454. doi:10.1007/ s12011-019-01793-5

4. Wu J, Pan X, Fu H, et al. Effect of curcumin on glycerol-induced acute kidney injury in rats. Sci Rep. 2017;7(1):1-11. doi:10.1038/ s41598-016-0028-x

5. Abd-Ellatif RN, Hegab II, Atef MM, Sadek MT, Hafez YM. Diacerein protects against glycerol-induced acute kidney injury: modulating oxidative stress, inflammation, apoptosis and necroptosis. Chem Biol Interact. 2019;306:47-53. doi:10.1016/j. cbi.2019.04.008

6. Reis NG, Francescato HDC, de Almeida LF, da Silva CGA, Costa RS, Coimbra TM. Protective effect of calcitriol on rhabdomyolysis-induced acute kidney injury in rats. Sci Rep. 2019;9(1):1-10. doi:10.1038/s41598-019-43564-1

7. Li YF, Xu BY, An R, et al. Protective effect of anisodamine in rats with glycerol-induced acute kidney injury. BMC Nephrol. 2019;20 (1):223. doi:10.1186/s12882-019-1394-y

8. Al Asmari AK, Al Sadoon KT, Obaid AA, Yesunayagam D, Tariq M. Protective effect of quinacrine against glycerol-induced acute kidney injury in rats. BMC Nephrol. 2017;18(1):41. doi:10.1186/s12882017-0450-8

9. Linkermann A, Chen G, Dong G, Kunzendorf U, Krautwald S, Dong Z. Regulated cell death in AKI. J Am Soc Nephrol. 2014;25 (12):2689-2701. doi:10.1681/ASN.2014030262

10. Wang S, Zhang C, Hu L, Yang C. Necroptosis in acute kidney injury: a shedding light. Cell Death Dis. 2016;7(3):e2125. doi:10.1038/ cddis. 2016.37

11. Zhang L, Jiang F, Chen Y, et al. Necrostatin-1 attenuates ischemia injury induced cell death in rat tubular cell line NRK-52E through decreased Drp1 expression. Int J Mol Sci. 2013;14(12):24742-24754. doi:10.3390/ijms 141224742

12. Linkermann A, Bräsen JH, Darding M, et al. Two independent pathways of regulated necrosis mediate ischemia-reperfusion injury. Proc Natl Acad Sci. 2013;110(29):12024-12029. doi:10.1073/pnas.1305538110

13. Guo LP, Liu SX, Yang Q, et al. Effect of thymoquinone on acute kidney injury induced by sepsis in BALB/c mice. Biomed Res Int. 2020;2020:1594726.

14. Ramadan SS, Almeer R, Albasher G, Abdel Moneim AE. Lycopene mitigates arsenic-induced nephrotoxicity with activation of the Nrf2 pathway in mice. Toxin Rev. 2021;1-11. doi:10.1080/15569543.2021.1891938

15. Stojiljkovic N, Ilic S, Jakovljevic V, et al. The encapsulation of lycopene in nanoliposomes enhances its protective potential in methotrexate-induced kidney injury model. Oxid Med Cell Longev. 2018;2018:1-11. doi:10.1155/2018/2627917

16. Augusti PR, Conterato GM, Somacal S, et al. Effect of lycopene on nephrotoxicity induced by mercuric chloride in rats. Basic Clin Pharmacol Toxicol. 2007;100(6):398-402. doi:10.1111/j.17427843.2007.00067.x

17. Erman F, Tuzcu M, Orhan C, Sahin N, Sahin K. Effect of lycopene against cisplatin-induced acute renal injury in rats: organic anion and cation transporters evaluation. Biol Trace Elem Res. 2014;158 (1):90-95. doi:10.1007/s12011-014-9914-x 
18. Bayomy NA, Elbakary RH, Ibrahim MAA, Abdelaziz EZ. Effect of lycopene and rosmarinic acid on gentamicin induced renal cortical oxidative stress, apoptosis, and autophagy in adult male albino rat. Anat Rec. 2017;300(6):1137-1149. doi:10.1002/ar.23525

19. Dai C, Tang S, Deng S, et al. Lycopene attenuates colistin-induced nephrotoxicity in mice via activation of the $\mathrm{Nrf2/HO}-1$ pathway. Antimicrob Agents Chemother. 2015;59(1):579-585. doi:10.1128/ AAC.03925-14

20. Guo Y, Liu Y, Wang Y. Beneficial effect of lycopene on anti-diabetic nephropathy through diminishing inflammatory response and oxidative stress. Food Funct. 2015;6(4):1150-1156. doi:10.1039/ C5FO00004A

21. Pierine DT, Navarro ME, Minatel IO, et al. Lycopene supplementation reduces TNF- $\alpha$ via RAGE in the kidney of obese rats. Nutr Diabetes. 2014;4(11):e142. doi:10.1038/nutd.2014.39

22. Liu L, Liu C, Hou L, et al. Protection against ischemia/reperfusion-induced renal injury by co-treatment with erythropoietin and sodium selenite. Mol Med Rep. 2015;12(6):7933-7940. doi:10.3892/ mmr.2015.4426

23. Sowndarya P, Ramkumar G, Shivakumar M. Green synthesis of selenium nanoparticles conjugated Clausena dentata plant leaf extract and their insecticidal potential against mosquito vectors. Artif Cells, Nanomed Biotechnol. 2017;45(8):1490-1495. doi:10.1080/ 21691401.2016.1252383

24. Krishnan V, Loganathan C, Thayumanavan P. Green synthesized selenium nanoparticles using Spermacoce hispida as carrier of s-allyl glutathione: to accomplish hepatoprotective and nephroprotective activity against acetaminophen toxicity. Artif Cells, Nanomed Biotechnol. 2019;47(1):56-63. doi:10.1080/21691401.2018.1543192

25. Zhang W, Zhang J, Ding D, et al. Synthesis and antioxidant properties of Lycium barbarum polysaccharides capped selenium nanoparticles using tea extract. Artif Cells, Nanomed Biotechnol. 2018;46 (7):1463-1470. doi:10.1080/21691401.2017.1373657

26. Ramamurthy C, Sampath KS, Arunkumar P, et al. Green synthesis and characterization of selenium nanoparticles and its augmented cytotoxicity with doxorubicin on cancer cells. Bioprocess Biosyst Eng. 2013;36(8):1131-1139. doi:10.1007/s00449-012-0867-1

27. Wang L, Li C, Huang Q, Fu X. Biofunctionalization of selenium nanoparticles with a polysaccharide from Rosa roxburghii fruit and their protective effect against $\mathrm{H}(2) \mathrm{O}(2)$-induced apoptosis in INS-1 cells. Food Funct. 2019;10(2):539-553. doi:10.1039/C8FO01958D

28. Kim JH, Lee SS, Jung MH, et al. N-acetylcysteine attenuates glycerol-induced acute kidney injury by regulating MAPKs and Bcl-2 family proteins. Nephrol Dial Transplant. 2010;25 (5):1435-1443. doi:10.1093/ndt/gfp659

29. Almeer RS, AlBasher GI, Alarifi S, Alkahtani S, Ali D, Abdel Moneim AE. Royal jelly attenuates cadmium-induced nephrotoxicity in male mice. Sci Rep. 2019;9(1):5825. doi:10.1038/s41598-01942368-7

30. Lowry OH, Rosebrough NJ, Farr AL, Randall RJ. Protein measurement with the Folin phenol reagent. J Biol Chem. 1951;193 (1):265-275. doi:10.1016/S0021-9258(19)52451-6

31. Ohkawa H, Ohishi N, Yagi K. Assay for lipid peroxides in animal tissues by thiobarbituric acid reaction. Anal Biochem. 1979;95 (2):351-358. doi:10.1016/0003-2697(79)90738-3

32. Green LC, Wagner DA, Glogowski J, Skipper PL, Wishnok JS, Tannenbaum SR. Analysis of nitrate, nitrite, and $[15 \mathrm{~N}]$ nitrate in biological fluids. Anal Biochem. 1982;126(1):131-138. doi:10.1016/ 0003-2697(82)90118-X

33. Ellman GL. Tissue sulfhydryl groups. Arch Biochem Biophys. 1959;82(1):70-77. doi:10.1016/0003-9861(59)90090-6

34. Nishikimi M, Appaji N, Yagi K. The occurrence of superoxide anion in the reaction of reduced phenazine methosulfate and molecular oxygen. Biochem Biophys Res Commun. 1972;46(2):849-854. doi:10.1016/S0006-291X(72)80218-3

35. Aebi H. Catalase in vitro. Methods Enzymol. 1984;105:121-126.
36. Paglia DE, Valentine WN. Studies on the quantitative and qualitative characterization of erythrocyte glutathione peroxidase. J Lab Clin Med. 1967;70(1):158-169.

37. De Vega L, Fernandez RP, Mateo MC, Bustamante JB, Herrero AM, Munguira EB. Glutathione determination and a study of the activity of glutathione-peroxidase, glutathione-transferase, and glutathione-reductase in renal transplants. Ren Fail. 2002;24 (4):421-432. doi:10.1081/JDI-120006769

38. Livak KJ, Schmittgen TD. Analysis of relative gene expression data using real-time quantitative PCR and the 2(-Delta Delta C(T)) method. Methods. 2001;25(4):402-408. doi:10.1006/meth.2001.1262

39. Alagesan V, Venugopal S. Green synthesis of selenium nanoparticle using leaves extract of withania somnifera and its biological applications and photocatalytic activities. BioNanoScience. 2019;9 (1):105-116. doi:10.1007/s12668-018-0566-8

40. Rajkumar K, Mvs S, Koganti S, Burgula S. Selenium nanoparticles synthesized using pseudomonas stutzeri (MH191156) show antiproliferative and anti-angiogenic activity against cervical cancer cells. Int J Nanomedicine. 2020;15:4523-4540. doi:10.2147/IJN.S247426

41. Qiao L, Dou X, Yan S, Zhang B, Xu C. Biogenic selenium nanoparticles synthesized by Lactobacillus casei ATCC 393 alleviate diquat-induced intestinal barrier dysfunction in C57BL/6 mice through their antioxidant activity. Food Funct. 2020;11 (4):3020-3031. doi:10.1039/D0FO00132E

42. Siddiqui RA, Simjee SU, Kabir N, Ateeq M, Shah MR, Hussain SS. $\mathrm{N}$-(2-hydroxyphenyl)acetamide and its gold nanoparticle conjugation prevent glycerol-induced acute kidney injury by attenuating inflammation and oxidative injury in mice. Mol Cell Biochem. 2019;450(12):43-52. doi:10.1007/s11010-018-3371-3

43. Sharawy MH, Abdelrahman RS, El-Kashef DH. Agmatine attenuates rhabdomyolysis-induced acute kidney injury in rats in a dose dependent manner. Life Sci. 2018;208:79-86. doi:10.1016/j.lfs.2018.07.019

44. Tsitsimpikou C, Kioukia-Fougia N, Tsarouhas $\mathrm{K}$, et al. Administration of tomato juice ameliorates lactate dehydrogenase and creatinine kinase responses to anaerobic training. Food Chem Toxicol. 2013;61:9-13. doi:10.1016/j.fct.2012.12.023

45. Li X, Wang Q, Deng G, et al. Porous Se@ SiO2 nanospheres attenuate cisplatin-induced acute kidney injury via activation of Sirt1. Toxicol Appl Pharmacol. 2019;380:114704. doi:10.1016/j. taap.2019.114704

46. Randjelovic P, Veljkovic S, Stojiljkovic N, et al. Protective effect of selenium on gentamicin-induced oxidative stress and nephrotoxicity in rats. Drug Chem Toxicol. 2012;35(2):141-148. doi:10.3109/ 01480545.2011.589446

47. Quagliariello V, Vecchione R, Coppola C, et al. Cardioprotective effects of nanoemulsions loaded with anti-inflammatory nutraceuticals against doxorubicin-induced cardiotoxicity. Nutrients. 2018;10 (9):1304. doi:10.3390/nu10091304

48. Zhao W, Huang X, Zhang L, et al. Penehyclidine hydrochloride pretreatment ameliorates rhabdomyolysis-induced AKI by activating the Nrf2/HO-1 pathway and alleviating endoplasmic reticulum stress in rats. PLoS One. 2016;11(3):e0151158. doi:10.1371/journal. pone. 0151158

49. Albarakati AJA, Baty RS, Aljoudi AM. et al. Luteolin protects against lead acetate-induced nephrotoxicity through antioxidant, anti-inflammatory, anti-apoptotic, and $\mathrm{Nrf2/HO}-1$ signaling pathways. Mol Biol Rep. 2020:1-13. doi:10.1007/s11033-019-04608-x

50. Yu K, Zhang J, Cao Z, et al. Lycopene attenuates AFB 1-induced renal injury with the activation of the Nrf2 antioxidant signaling pathway in mice. Food Funct. 2018;9(12):6427-6434. doi:10.1039/ C8FO01301B

51. Zhu C, Zhang S, Song C, et al. Selenium nanoparticles decorated with Ulva lactuca polysaccharide potentially attenuate colitis by inhibiting NF- $\mathrm{kB}$ mediated hyper inflammation. $J$ Nanobiotechnology. 2017;15(1):1-15. doi:10.1186/s12951-0170252-y 
52. Guerrero-Hue M, García-Caballero C, Palomino-Antolín A, et al Curcumin reduces renal damage associated with rhabdomyolysis by decreasing ferroptosis-mediated cell death. FASEB J. 2019;33 (8):8961-8975. doi:10.1096/fj.201900077R

53. Dogukan A, Tuzcu M, Agca CA, et al. A tomato lycopene complex protects the kidney from cisplatin-induced injury via affecting oxidative stress as well as Bax, Bcl-2, and HSPs expression. Nutr Cancer. 2011;63(3):427-434. doi:10.1080/01635581.2011.535958
54. Çevik Ö, Oba R, Macit Ç, et al. Lycopene inhibits caspase-3 activity and reduces oxidative organ damage in a rat model of thermal injury. Burns. 2012;38(6):861-871. doi:10.1016/j.burns.2012.01.006

55. Homsi E, Andreazzi DD, Faria JBLD, Janino P. TNF- $\alpha$-mediated cardiorenal injury after rhabdomyolysis in rats. Am J Physiol Renal Physiol. 2015;308(11):F1259-F1267. doi:10.1152/ajprenal.00311. 2014

\section{Publish your work in this journal}

The International Journal of Nanomedicine is an international, peerreviewed journal focusing on the application of nanotechnology in diagnostics, therapeutics, and drug delivery systems throughout the biomedical field. This journal is indexed on PubMed Central, MedLine, CAS, SciSearch ${ }^{\mathbb{B}}$, Current Contents ${ }^{\mathbb{B}} /$ Clinical Medicine, $^{2}$
Journal Citation Reports/Science Edition, EMBase, Scopus and the Elsevier Bibliographic databases. The manuscript management system is completely online and includes a very quick and fair peer-review system, which is all easy to use. Visit http://www.dovepress.com/ testimonials.php to read real quotes from published authors. 